1 Food Chemistry (Research paper)

2

\title{
3 Oligomerization mechanism of tea catechins during tea roasting
}

4

5 Hitomi Morikawa ${ }^{a}$, Keita Okuda ${ }^{a}$, Yuji Kunihira ${ }^{a}$, Aoi Inada ${ }^{a}$, Chika Miyagi ${ }^{b}$, Yosuke 6 Matsuo $^{\text {a }}$, Yoshinori Saito ${ }^{\text {a }}$, Takashi Tanaka ${ }^{\mathrm{a}, *}$

7

a Department of Natural Product Chemistry, Graduate School of Biomedical Sciences,

$9 \quad$ Nagasaki University, 1-14 Bunkyo-machi, Nagasaki 852-8521, Japan

10

b Department of Natural Product Chemistry, School of Pharmaceutical Sciences,

* Corresponding author: Department of Natural Product Chemistry, Graduate School of Biomedical Sciences, Nagasaki University, 1-14 Bunkyo-machi, Nagasaki 852-8521, Japan, Tel: +8195 8192433, E-mail address: t-tanaka@nagasaki-u.ac.jp (T. Tanaka) 
17 Abstract: Roasting of green tea causes oligomerization of tea catechins, which 18 decreases the astringency. The aim of this study was to elucidate the oligomerization 19 mechanism. The ${ }^{13} \mathrm{C}$ NMR spectrum of the oligomer fraction showed signals arising 20 from catechin and sugar residues. Heating of epigallocatechin-3-O-gallate with

$21{ }^{13} \mathrm{C}$-labeled glucose $\left(150^{\circ} \mathrm{C}\right.$ for $\left.2 \mathrm{~h}\right)$ suggested that condensation of sugars with catechin 22 A-rings caused the oligomerization. The dimeric product obtained by heating for a 23 shorter period (30 min) suggested cross-linking occurred between sugars and catechin 24 A-rings. Furthermore, heating of phloroglucinol, a catechin A-ring mimic, with glucose, 25 methylglyoxal, and dihydroxyacetone, confirmed that the basic mechanism included 26 reaction of the catechin A-ring methine carbons with carbonyl carbons of glucose and 27 their pyrolysis products.

29 Keywords: roasted tea; catechin; sugar; methylglyoxal; dihydroxyacetone; 30 phloroglucinol; polyphenol 


\section{Introduction}

Roasting is an important method for processing, cooking, and preserving foods, and is essential in the production of cocoa and coffee as it add characteristic aromas and flavors. However, chemical changes occur in phenolic substances during the roasting process and these changes are complex and not clearly understood (Taeye, Bodart, Caullet, \& Collin, 2017; Moreira, et al., 2017). In Japan, roasted green tea is a popular processed tea, and it is served in traditional Japanese multi-course dinners for the elderly. Roasting adds characteristic flavor via caramelization and Maillard reactions and decreases the caffeine content by sublimation, which is beneficial to reduce the effect of tea on sleep. In addition, roasted green tea has a milder taste than non-roasted green tea, and this is thought to be caused by a decrease in the content of astringent tea catechins. A comparison of HPLC profiles of an original green tea product and a roasted green tea (Fig. 1) showed roasting resulted in epimerization at the catechin C-2 position (Suzuki, et al., 2003; Seto, Nakamura, Nanjo, \& Hara, 1997) and generation of oligomeric products, which were detected as a broad hump on the baseline. Astringency is caused by interactions with salivary proteins (Haslam, 1996; Baxter, Lilley, Haslam, \& Williamson, 1997); thus, the epimerization products (1a-4a) do not contribute to the decrease in astringency because the affinities of 2,3-trans epimerization products in human serum albumin are stronger than in the original 2,3-cis tea catechins (Ishii et al., 2010). Therefore, oligomerization of tea catechins is mainly responsible for the decrease in tea astringency with roasting; however, the chemical mechanisms for this are unknown. The aim of this study was to elucidate the oligomerization mechanism using model experiments and spectroscopic methods. 


\subsection{Materials}

Green tea leaves were supplied by Nagasaki Agriculture and Forestry Technical

Development Center, Higashisonogi Tea Research Station (Nagasaki, Japan). $1-{ }^{13} \mathrm{C}$-D-Glucose and $\mathrm{U}_{-}{ }^{13} \mathrm{C}_{6}$-D-glucose were purchased from Cambridge Isotope Laboratories, Inc. (MA, USA). Epigallocatechin-3-O-gallate was isolated from commercial green tea according to a reported method (Nonaka, Kawahara, \& Nishioka, 1983).

\subsection{Analytical procedures}

Ultraviolet (UV)-visible spectra were obtained using a JASCO V-560 spectrophotometer (Jasco Co., Tokyo, Japan). ${ }^{1} \mathrm{H}$ and ${ }^{13} \mathrm{C}$ NMR spectra were recorded in acetone- $d_{6}, \mathrm{CD}_{3} \mathrm{OD}$, and DMSO- $d_{6}$ (Kanto Chemical Co. Inc., Tokyo, Japan) at $27^{\circ} \mathrm{C}$ with a JEOL JNM-AL400 spectrometer (JEOL Ltd., Tokyo, Japan) operating at 400 and $100 \mathrm{MHz}$ for ${ }^{1} \mathrm{H}$ and ${ }^{13} \mathrm{C}$ nuclei, respectively. The coupling constants are expressed in hertz, and chemical shifts are presented on the delta (ppm) scale. Column chromatography was performed using Sephadex LH-20 (25-100 $\mu \mathrm{m}$, GE Healthcare Bio-Science AB, Uppsala), Diaion HP20SS (Mitsubishi Chemical, Japan), MCI-gel CHP 20P (75-150 $\mu \mathrm{m}$; Mitsubishi Chemical, Tokyo, Japan), Chromatorex ODS (100-200 mesh; Fuji Silysia Chemical, Kasugai, Japan), and silica gel 60N (100-250 $\mu \mathrm{m}$, Kanto Chemical Co., Tokyo, Japan) columns. Thin layer chromatography (TLC) was performed on precoated Kieselgel $60 \mathrm{~F}_{254}$ plates $(0.2-\mathrm{mm}$ thick, Merck KGaA, Darmstadt, Germany) with toluene:ethyl formate:formic acid (1:7:1, v/v/v) and $\mathrm{CHCl}_{3}: \mathrm{MeOH}$ :water $(14: 6: 1, \mathrm{v} / \mathrm{v} / \mathrm{v})$. Spots were detected under UV illumination after 
spraying with $2 \%$ ethanolic $\mathrm{FeCl}_{3}$ or a $5 \%$ sulfuric acid reagent and heating. Analytical HPLC was performed using a Cosmosil 5C18-AR II (Nacalai Tesque Inc., Kyoto, Japan) column (4.6 mm i.d. $\times 250 \mathrm{~mm}$ ) with a gradient elution from $4 \%-30 \% \mathrm{CH}_{3} \mathrm{CN}(39 \mathrm{~min})$ and $30 \%-75 \% \mathrm{CH}_{3} \mathrm{CN}\left(15 \mathrm{~min}\right.$ ) in $50 \mathrm{mM} \mathrm{H}_{3} \mathrm{PO}_{4}$. The mobile phase flow rate was 0.8 $\mathrm{mL} / \mathrm{min}$, and detection was achieved using a Jasco MD-910 photodiode array detector.

\subsection{Heating of (-)-epigallocatechin-3-O-gallate (2)}

An aqueous solution $(200 \mathrm{~mL})$ of (-)-epigallocatechin-3-O-gallate $(2)(1.0 \mathrm{~g})$ was lyophilized, and the resulting white powder was heated at $150^{\circ} \mathrm{C}$ for $60 \mathrm{~min}$ in an electric furnace (Yamato Scientific co., ltd., Tokyo, Japan). The reaction mixture was subjected to Sephadex LH-20 column chromatography $(3 \mathrm{~cm}$ i.d. $\times 25 \mathrm{~cm})$ with $0-100 \% \mathrm{MeOH}$ containing $1 \%$ trifluoroacetic acid (10\% stepwise, each $100 \mathrm{~mL})$ to produce (-)-gallocatechin-3-O-gallate $(142.5 \mathrm{mg})$, gallic acid $(18.2 \mathrm{mg})$, and tricetinidin (12.2 mg) (Kuhnert N., Clifford M. N., \& Radenac A-G., 2001; Coggon, Moss, Graham, \& Sanderson, 1973) and recover 2 (756 mg).

\subsection{Roasting of green tea leaves and separation of oligomeric polyphenols}

Green tea leaves $(20 \mathrm{~g})$ were heated at $180^{\circ} \mathrm{C}$ in an electric furnace for $30 \mathrm{~min}$. After cooling, the leaves were extracted twice with acetone: $\mathrm{H}_{2} \mathrm{O}(3: 2, \mathrm{v} / \mathrm{v}, 300 \mathrm{~mL})$ at r.t. The extract was concentrated using a rotary evaporator, and the resulting aqueous solution $(150 \mathrm{~mL})$ was partitioned with EtOAc $(150 \mathrm{~mL})$ twice to produce an EtOAc fraction $(2.97 \mathrm{~g})$. The aqueous layer was first concentrated to remove residual EtOAc and then subjected to Diaion HP20SS column chromatography $(3 \mathrm{~cm}$ i.d. $\times 20 \mathrm{~cm})$ with $\mathrm{H}_{2} \mathrm{O}: \mathrm{MeOH}(0-100 \%, 20 \%$ stepwise, each $100 \mathrm{~mL})$. The eluate was monitored by TLC 
and separated into two fractions (Fr.). Fraction 1 mainly contained sugars (3.1 g), and Fr. 2 contained catechins and caffeine $(2.1 \mathrm{~g})$. Fraction 2 was subjected to size-exclusion column chromatography using Sephadex LH-20 $(2 \mathrm{~cm}$ i.d. $\times 55 \mathrm{~cm})$ with $7 \mathrm{M}$ urea:acetone (2:3, v/v, containing conc. $\mathrm{HCl}$ at $5 \mathrm{~mL} / \mathrm{L}$ ) (Yanagida, Shoji, \& Shibusawa, on the baseline in HPLC analysis (Fig. S1).

\subsection{Heating of (-)-epigallocatechin-3-O-gallate (2) with sucrose}

$(1.0 \mathrm{~g})$ were dissolved in $\mathrm{H}_{2} \mathrm{O}(10 \mathrm{~mL})$ and heated on a hot plate until most of the water evaporated. The resulting paste was heated at $150^{\circ} \mathrm{C}$ for $2 \mathrm{~h}$ in an electric furnace, and the products were separated by Sephadex LH-20 column chromatography $(3 \mathrm{~cm}$ i.d. $\times 12 \mathrm{~cm})$ with 0-100\% $\mathrm{MeOH}\left(20 \%\right.$ stepwise, each $100 \mathrm{~mL}$ ) and then $\mathrm{MeOH}: \mathrm{H}_{2} \mathrm{O}$ :acetone (60:20:20 and $0: 1: 1 \mathrm{v} / \mathrm{v} / \mathrm{v}$ steps, each $100 \mathrm{~mL}$ ) to produce two fractions. The first fraction was purified by Diaion HP20SS column chromatography $(3 \mathrm{~cm}$ i.d. $\times 12 \mathrm{~cm})$ with $0-100 \%$ $\mathrm{MeOH}(10 \%$ stepwise, each $100 \mathrm{~mL})$ to produce the pure oligomers $(307 \mathrm{mg})$. The second fraction (1.16 g) also contained oligomers as the major constituents as well as gallic acid and minor impurities (Fig. S2). dissolved in $\mathrm{H}_{2} \mathrm{O}$ :acetone $(4: 1, \mathrm{v} / \mathrm{v}, 2.5 \mathrm{~mL})$ in a petri dish and concentrated by heating at $75^{\circ} \mathrm{C}$. The resulting paste was heated at $150^{\circ} \mathrm{C}$ for $2 \mathrm{~h}$ in the electric furnace, and the 
products were separated by Sephadex LH-20 column chromatography $(2 \mathrm{~cm}$ i.d. $\times 25$ $\mathrm{cm})$ with $60 \%-100 \% \quad \mathrm{MeOH} \quad(20 \%$ stepwise, each $100 \mathrm{~mL})$ and then $\mathrm{MeOH}-\mathrm{H}_{2} \mathrm{O}-$ acetone $(90: 5: 5,80: 10: 10,60: 20: 20$, and 0:1:1 v/v/v steps, each $100 \mathrm{~mL}$ ) to give five fractions: Fr. 1 (172 mg), Fr. 2 (186 mg), Fr. 3 (290.4 mg), Fr. 4 (77 mg), and Fr. 5 (192 mg). HPLC of Fr. 5 showed that it only contained oligomeric products.

\subsection{Heating of (-)-epigallocatechin-3-O-gallate (2) with ${ }^{13} \mathrm{C}$-labeled glucose} Similarly, 2 (510 mg) was heated with a 9:1 mixture of glucose and $1-{ }^{13} \mathrm{C}$-D-glucose (total of $386 \mathrm{mg}$ ) to yield the oligomers (182 mg). Experiments using 2 (510 mg) and a 9:1 mixture of glucose and $\mathrm{U}^{13} \mathrm{C}$-D-glucose (total of $386 \mathrm{mg}$ ) afforded the oligomers $(190 \mathrm{mg})$.

\subsection{Monomeric and dimeric products of $\mathbf{2}$ produced by heating with glucose} An aqueous solution $(400 \mathrm{~mL})$ of $2(2.0 \mathrm{~g})$ and D-glucose $(1.6 \mathrm{~g})$ was lyophilized, and the resulting white powder was heated at $150^{\circ} \mathrm{C}$ for $30 \mathrm{~min}$. The reaction mixture was subjected to MCI-gel CHP20P column chromatography $(3 \mathrm{~cm}$ i.d. $\times 20 \mathrm{~cm})$ with $0-100 \% \mathrm{MeOH}(10 \%$ stepwise, each $100 \mathrm{~mL})$ to give five fractions: Fr. 1 (773 mg), Fr. 2 (246 mg), Fr. 3 (654 mg), Fr. 4 (402 mg), and Fr. 5 (139 mg). Separation of Fr. 1 using the Sephadex LH-20 column $(2 \mathrm{~cm}$ i.d. $\times 20 \mathrm{~cm})$ with $0-100 \% \mathrm{MeOH}$ in $\mathrm{H}_{2} \mathrm{O}(10 \%$ stepwise, each $100 \mathrm{~mL})$ gave $5(32.2 \mathrm{mg}), 6(95.7 \mathrm{mg})$, and $7(12.3 \mathrm{mg})$. Fraction 3 was subjected to Sephadex LH-20 column chromatography $(3 \mathrm{~cm}$ i.d. $\times 20$ $\mathrm{cm})$ with $0-100 \% \mathrm{MeOH}(10 \%$ stepwise, each $100 \mathrm{~mL})$ to produce $2(93.8 \mathrm{mg})$ and $2 \mathrm{a}$ (71.9 mg). Fractions 4 and 5 mainly contained oligomeric products, and separation of Fr. 4 using the Sephadex LH-20 column $(3 \mathrm{~cm}$ i.d. $\times 20 \mathrm{~cm})$ with $20-100 \% \mathrm{MeOH}(10 \%$ 
stepwise, each $100 \mathrm{~mL}$ ) and subsequent purification using Chromatorex ODS column chromatography $(3 \mathrm{~cm}$ i.d. $\times 25 \mathrm{~cm})$ with $0-50 \% \mathrm{MeOH}(5 \%$ stepwise, each $100 \mathrm{~mL})$ furnished 8 (10.4 mg).

\subsubsection{8-C-Glucosyl-(-)-epigallocatechin-3-O-gallate (5)}

Brown amorphous powder, $[\alpha]_{\mathrm{D}}-195.4$ ( $c$ 0.10, MeOH). FAB-MS $m / z: 621$ $[\mathrm{M}+\mathrm{H}]^{+}, 643[\mathrm{M}+\mathrm{Na}]^{+}$. HR-FABMS m/z: 621.1465 (Calcd for $\mathrm{C}_{28} \mathrm{H}_{29} \mathrm{O}_{16}$ : 621.1456), 643.1293 (Calcd for $\mathrm{C}_{28} \mathrm{H}_{28} \mathrm{NaO}_{16}: 643.1275$ ). UV $\lambda_{\max } \mathrm{nm}(\log \varepsilon): 211$ (4.88), 276 (4.02). IR $v_{\max } \mathrm{cm}^{-1}: 3388,1691,1613,1537,1453,1448 .{ }^{1} \mathrm{H}$ NMR (acetone- $d_{6}+\mathrm{D}_{2} \mathrm{O}$ )

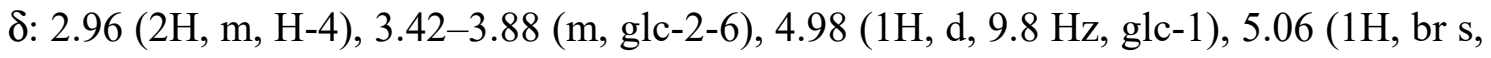
H-2), 5.34 (1H, m, H-3), 5.98 (1H, s, H-6), 6.75 (2H, s, B-2,6), 6.99 (2H, s, galloyl-H).

${ }^{13} \mathrm{C}$ NMR (acetone- $d_{6}+\mathrm{D}_{2} \mathrm{O}$ ) $\delta: 26.3$ (C-4), 61.6 (glc-6), 69.4 (C-3), 70.8 (glc-4), 73.5 (glc-2), 76.2 (glc-1), 77.9 (C-2), 78.7 (glc-3), 81.6 (glc-5), 96.8 (C-6), 98.4 (C-4a), 103.5 (C-8), 106.3 (B-ring-2,6), 109.8 (galloyl-2,6), 121.1 (galloyl-1), 130.6 (B-ring-1), 132.7 (B-ring-4), 138.7 (galloyl-4), 145.6 (galloyl-3,5), 146.0 (B-ring-3,5), 154.0 (C-8a), 156.3 (C-7), 157.0 (C-5), 166.4 (galloyl-7).

\subsubsection{6-C-Glucosyl-(-)-epigallocatechin-3-O-gallate (6)}

Brown amorphous powder, $[\alpha]_{\mathrm{D}}-66.6$ ( $c$ 0.10, MeOH). FAB-MS $m / z: 621$ $[\mathrm{M}+\mathrm{H}]^{+}, 643[\mathrm{M}+\mathrm{Na}]^{+}$. HR-FABMS m/z: 621.1451 (Calcd for $\mathrm{C}_{28} \mathrm{H}_{29} \mathrm{O}_{16}:$ 621.1456), 643.1279 (Calcd for $\mathrm{C}_{28} \mathrm{H}_{28} \mathrm{NaO}_{16}: 643.1275$ ). UV $\lambda_{\max } \mathrm{nm}(\log \varepsilon): 211$ (4.83), 276 (3.99). IR $v_{\max } \mathrm{cm}^{-1}: 3393,1681,1613,1536,1453,1337 .{ }^{1} \mathrm{H}$ NMR (acetone- $d_{6}+\mathrm{D}_{2} \mathrm{O}$ )

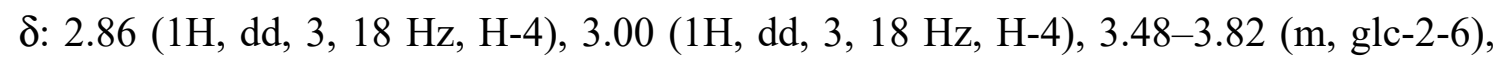
$4.83(1 \mathrm{H}, \mathrm{d}, 9.6 \mathrm{~Hz}, \mathrm{glc}-1), 5.01(1 \mathrm{H}$, br s, H-2), 5.43 (1H, m, H-3), 6.06 (1H, s, H-8), $6.61(2 \mathrm{H}, \mathrm{s}, \mathrm{B}-2,6), 6.99\left(2 \mathrm{H}, \mathrm{s}\right.$, galloyl-H). ${ }^{13} \mathrm{C}$ NMR (acetone- $\left.d_{6}+\mathrm{D}_{2} \mathrm{O}\right) \delta: 26.5(\mathrm{C}-4)$, 61.3 (glc-6), 69.5 (C-3), 70.1 (glc-4), 74.6 (glc-2), 76.8 (glc-1), 78.0 (C-2), 79.0 (glc-3), 
81.8 (glc-5), 96.6 (C-8), 100.3 (C-4a), 105.4 (C-6), 106.5 (B-ring-2,6), 109.8 (galloyl-2,6), 121.4 (galloyl-1), 130.3 (B-ring-1), 133.0 (B-ring-4), 138.8 (galloyl-4), 145.8 (galloyl-3,5), 146.0 (B-ring-3,5), 155.3 (C-7), 155.6 (C-5), 156.0 (C-8a), 166.5 (galloyl-7).

\subsubsection{6-C-Substituted product of (-)-epigallocatechin-3-O-gallate (7)}

Brown amorphous powder, $[\alpha]_{\mathrm{D}}-136.8$ (c $\left.0.12, \mathrm{MeOH}\right), \mathrm{UV}(\mathrm{MeOH}) \lambda_{\max }(\log$ ع): 268 (4.04), IR $v_{\max } \mathrm{cm}^{-1}: 3404,1693,1619,1537,1455,1339$. HR-ESI-MS $[\mathrm{M}+\mathrm{H}]^{+}$ $m / z: 765.1873[\mathrm{M}+\mathrm{H}]^{+}\left(\right.$Calcd for $\left.\mathrm{C}_{34} \mathrm{H}_{37} \mathrm{O}_{20}, 765.1873\right) .{ }^{1} \mathrm{H}$ NMR (acetone- $d_{6}+\mathrm{D}_{2} \mathrm{O}$ )

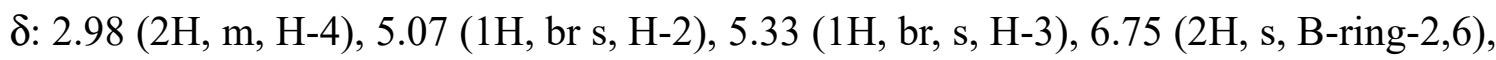
6.98 (2H, s, galloyl-2,6), C-6-sugar unit: $3.01(1 \mathrm{H}, \mathrm{d}, J=16.5 \mathrm{~Hz}, \mathrm{H}-1), 3.20(1 \mathrm{H}, \mathrm{d}, J$ $=16.5 \mathrm{~Hz}, \mathrm{H}-1), 3.63(2 \mathrm{H}, \mathrm{m}, \mathrm{H}-6), 3.89(1 \mathrm{H}, \mathrm{m}, \mathrm{H}-5), 4.05(1 \mathrm{H}, \mathrm{d}, J=8.3 \mathrm{~Hz}, \mathrm{H}-3)$, $4.16(1 \mathrm{H}, \mathrm{t}, J=8.3 \mathrm{~Hz}, \mathrm{H}-4), \mathrm{C}-8$-glucosyl: 3.45 (1H, m, H-5), 3.66 (m, H-4), 3.67 (m, H-3), 3.83 (m, H-2), $3.84(2 \mathrm{H}, \mathrm{m}, \mathrm{H}-6) .{ }^{13} \mathrm{C}$ NMR (acetone- $\left.d_{6}+\mathrm{D}_{2} \mathrm{O}\right) \delta: 26.0(\mathrm{C}-4)$, 69.2 (C-3), 78.1 (C-2), 94.8 (C-4a), 104.5 (C-6), 104.7 (C-8), 106.9 (B-ring-2,6), 109.8 (galloyl-2,6), 121.0 (galloyl-1), 130.4 (B-ring-1), 132.7 (B-ring-4), 138.8 (galloyl-4), 145.5 (B-ring-3,5), 146.0 (galloyl-3,5), 151.6 (C-7), 153.3 (C-5), 157.9 (C-8a), 166.4 (galloyl-7); C-6-sugar unit: 34.8 (C-1), 64.3 (C-6), 76.6 (C-4), 80.7 (C-3), 83.9 (C-5), 116.9 (C-2); C-8-glucosyl: 61.4 (C-6), 70.6 (C-4), 73.8 (C-3), 75.4 (C-1), 78.6 (C-2), $81.6(\mathrm{C}-5)$.

\subsubsection{Dimeric product (8)}

Brown amorphous powder, $[\alpha]_{\mathrm{D}}-168.6(c 0.10, \mathrm{MeOH}), \mathrm{UV}(\mathrm{MeOH}) \lambda_{\max }(\log$ ع): 211 (5.02), 269 (4.41), IR $v_{\max } \mathrm{cm}^{-1}: 3438,1697,1620,1537,1445$. HR-ESI-MS $[\mathrm{M}+\mathrm{Na}]^{+} m / z: 1065.1955[\mathrm{M}+\mathrm{Na}]^{+}\left(\mathrm{Calcd}\right.$ for $\left.\mathrm{C}_{50} \mathrm{H}_{42} \mathrm{NaO}_{25}: 1065.1913\right) .{ }^{1} \mathrm{H} \mathrm{NMR}$ (acetone- $d_{6}+\mathrm{D}_{2} \mathrm{O}$ ) $\delta: 2.98,3.09$ (each 2H, m, H-4, 4'), 5.06, 5.12 (each 1H, br s, H-2, 
2'), 5.51 (2H, br, s, H-3, 3'), 6.13, 6.51 (each 1H, s, A-ring-H), 6.67, 6.68 (each 2H, s, B-ring-2,6), 7.00, 7.12 (each 2H, s, galloyl-2,6), sugar unit: $3.68(1 \mathrm{H}, \mathrm{ddd}, J=2.8,7.1$, 9.2, 3.79, H-5), 3.79 (1H, dd, $J=7.1,11.4 \mathrm{~Hz}, \mathrm{H}-6), 4.01$ (1H, dd, $J=2.8,11.4 \mathrm{~Hz}$, H-6), $4.63(1 \mathrm{H}, \mathrm{dd}, J=3.0,9.2 \mathrm{~Hz}, \mathrm{H}-4), 5.39(1 \mathrm{H}, \mathrm{dd}, J=1.6,3.0 \mathrm{~Hz}, \mathrm{H}-3), 6.52(1 \mathrm{H}$, $\mathrm{d}, J=1.6 \mathrm{~Hz}, \mathrm{H}-2) .{ }^{13} \mathrm{C}$ NMR (acetone- $\left.d_{6}+\mathrm{D}_{2} \mathrm{O}\right) \delta: 27.1,27.3(\mathrm{C}-4,4$ ) $), 69.1,69.5(\mathrm{C}-3$, 3'), 78.1, 79.1 (C-2, 2'), 91.1, 98.2 (A-ring methine), 99.1, 101.9 (C-4a, 4a'), 102.9, 111.2 (A-ring C-6 or C-8), 106.5 (B-ring-2,6), 109.8, 109.9 (galloyl-2,6), 121.0, 121.3 (galloyl-1), 129.9, 130.2 (B-ring-1), 133.0, 133.2 (B-ring-4), 138.8, 139.0 (galloyl-4), 208 145.7, 145.8 (galloyl-3,5), 146.1, 146.2 (B-ring-3,5), 155.5 (C-8a), 155.3, 157.0 (C-5), 154.1, 156.6 (C-7), 166.1 (galloyl-7); C-6-sugar unit: 38.1 (C-3), 64.3 (C-6), 74.0 (C-4), 74.4 (C-5), 101.7 (C-2), 148.2 (C-1).

211

\subsection{Reaction of phloroglucinol and glucose}

g) and D-glucose (4.5

g) were dissolved in dimethyl formamide $(5 \mathrm{~mL})$ and heated at $80^{\circ} \mathrm{C}$ for $24 \mathrm{~h}$. The mixture was poured into $\mathrm{H}_{2} \mathrm{O}(100$ $\mathrm{mL})$, and the resulting phloroglucinol precipitate $(2.0 \mathrm{~g})$ was removed by filtration. The filtrate was applied to a Chromatorex ODS column $(3 \mathrm{~cm}$ i.d. $\times 25 \mathrm{~cm})$ with $0-100 \%$ $\mathrm{MeOH}(10 \%$ stepwise, each $100 \mathrm{~mL})$ to yield Fr. 1 and $\mathbf{1 0}(227 \mathrm{mg})$. Fraction 1 was separated using Sephadex LH-20 column chromatography $(4 \mathrm{~cm}$ i.d. $\times 30 \mathrm{~cm})$ with $0-100 \% \mathrm{MeOH}(20 \%$ stepwise, each $200 \mathrm{~mL}$ ) to give glucose, Fr. 1-1, and Fr. 1-2, and recover phloroglucinol (1.7 g). Fraction 1-1 was purified via silica gel column chromatography $\left(\mathrm{CHCl}_{3}: \mathrm{MeOH}: \mathrm{H}_{2} \mathrm{O}, 70: 30: 5\right.$, and $60: 40: 10 \mathrm{v} / \mathrm{v} / \mathrm{v}$ steps) to yield 9 (378.7 mg). Similar silica gel column chromatography of Fr. 1-2 yielded an inseparable mixture of stereoisomers of $\mathbf{1 1}(346.7 \mathrm{mg})$. Treatment of $\mathbf{1 1}(40 \mathrm{mg})$ with $\mathrm{Ac}_{2} \mathrm{O}(0.8$ 
$\mathrm{mL})$ in pyridine $(0.5 \mathrm{~mL})$ at r.t. for $10 \mathrm{~h}$ and subsequent silica gel column

chromatography with hexane:acetone $(3: 2, \mathrm{v} / \mathrm{v})$ afforded an acetate of one of the

226

227

228

229

230

231

232

233

234

235

236

237

238

239

240

241

242

243

244

245

246

247 isomers of $\mathbf{1 1}$ (47 mg).

2.9.1. Phloroglucinol-C- $\beta$-D-glucoside (9)

White amorphous powder. $[\alpha]_{\mathrm{D}}+37.9$ ( $c$ $\left.0.1, \mathrm{MeOH}\right)$. FAB-MS $m / z: 289$ $[\mathrm{M}+\mathrm{H}]^{+}$. HR-FABMS $m / z: 289.0923$ (Calcd for $\mathrm{C}_{12} \mathrm{H}_{17} \mathrm{O}_{8}:$ 289.0918). IR $v_{\max } \mathrm{cm}^{-1}$ : 3373, 1620, 1452, 1148, 1040. ${ }^{1} \mathrm{H}$ NMR (400 MHz, CD $\left.3 \mathrm{OD}\right) \delta: 3.37$ (1H, m, glc-5), $3.46(2 \mathrm{H}, \mathrm{m}, \mathrm{glc}-6), 3.74(1 \mathrm{H}, \mathrm{dd}, J=12.1,4.8 \mathrm{~Hz}$ glc-4), $3.83(1 \mathrm{H}, \mathrm{dd}, J=9.8,4.6 \mathrm{~Hz}$, glc-2), $3.86(1 \mathrm{H}, \mathrm{brd}, J=4.6 \mathrm{~Hz}$, glc-3), $4.79(1 \mathrm{H}, \mathrm{d}, J=9.8 \mathrm{~Hz}, \mathrm{glc}-1), 5.86(2 \mathrm{H}, \mathrm{s}$, H-3, 4). ${ }^{13} \mathrm{C}$ NMR (100 MHz, CD 3 OD) $\delta: 62.4$ (glc-6), 71.4 (glc-4), 73.8 (glc-2), 76.8 (glc-1), 79.9 (glc-3), 82.4 (glc-5), 96.4 (C-3, 5), 104.3 (C-1), 158.9 (C-2, 6), 159.6 (C-4).

\subsubsection{Dimeric product 10}

Yellow amorphous powder. $[\alpha]_{\mathrm{D}}-24.4$ ( c $\left.0.1, \mathrm{MeOH}\right)$. FAB-MS $m / z: 397$ $[\mathrm{M}+\mathrm{H}]^{+}$. HR-FABMS $m / z$ : 397.1145 (Calcd for $\mathrm{C}_{18} \mathrm{H}_{21} \mathrm{O}_{10}$ : 397.1134$)$. UV $\lambda_{\max } \mathrm{nm}(\log$ ع): 216 (4.69). IR $v_{\max } \mathrm{cm}^{-1}: 3373,1620,1452,1148,1040 .{ }^{1} \mathrm{H}$ NMR (400 MHz, $\left.\mathrm{CD}_{3} \mathrm{OD}\right) \delta: 3.48(1 \mathrm{H}, \mathrm{dd}, J=5.8,13.6 \mathrm{~Hz}$, sug-6), $3.62(1 \mathrm{H}, \mathrm{dd}, J=3.4,13.6 \mathrm{~Hz}$, sug-6), $3.51(1 \mathrm{H}, \mathrm{dd}, J=2.3,7.3 \mathrm{~Hz}$, sug-4), $3.59(1 \mathrm{H}, \mathrm{ddd}, J=3.4,5.8,7.3 \mathrm{~Hz}$, sug-5), 3.74 $(1 \mathrm{H}, \mathrm{dd}, J=2.3,3.2 \mathrm{~Hz}$, sug-3), $3.82(1 \mathrm{H}, \mathrm{dd}, J=3.2,6.5 \mathrm{~Hz}$, sug-2), $4.53(1 \mathrm{H}, \mathrm{d}, J=.5$ Hz, sug-1), 6.07 (1H, d, $\left.J=2.3 \mathrm{~Hz}, \mathrm{H}-5^{\prime}\right), 6.08(1 \mathrm{H}, \mathrm{d}, J=2.3 \mathrm{~Hz}, \mathrm{H}-3$ '), $6.11(1 \mathrm{H}, \mathrm{d}, J$ $\left.=2.3 \mathrm{~Hz}, \mathrm{H}-3^{\prime}\right), 6.12\left(1 \mathrm{H}, \mathrm{d}, J=2.3 \mathrm{~Hz}, \mathrm{H}-3^{\prime}\right) .{ }^{13} \mathrm{C} \mathrm{NMR}\left(100 \mathrm{MHz}, \mathrm{CD}_{3} \mathrm{OD}\right) \delta: 33.5$ (glc-1), 64.5 (glc-6), 70.1 (glc-3), 73.0 (glc-5), 75.3 (glc-4), 79.4 (glc-2), 99.4 (C-3), 98.9 (C-5), 96.3 (C-3'), 96.7 (C-7'), 103.9 (2C, C-1, C-1'), 156.1 (C-2'), 156.2 (C-6'), 156.9 (C-2), 157.1 (C-6), 158.4 (C-4'), 158.5 (C-4). 


\subsubsection{Acetate of bisphloroglucinolyl glucose (11)}

White amorphous powder; $[\alpha]_{\mathrm{D}}+112.2(c 0.11, \mathrm{MeOH})$; UV $\lambda \max \mathrm{nm}(\log \varepsilon)$ : 280 (0.15), 206 (1.80); IR $v_{\max } \mathrm{cm}^{-1}: 3453,1748,1211$; FAB-MS (positive, matrix; m-nitrobenzyl alcohol) m/z: $775[\mathrm{M}+\mathrm{H}]^{+}$; HR-FAB-MS m/z: $775.2088[\mathrm{M}+\mathrm{H}]^{+}(\mathrm{Calcd}$ for $\mathrm{C}_{36} \mathrm{H}_{39} \mathrm{O}_{19}: 775.2086$ ); ${ }^{1} \mathrm{H}$ NMR (acetone- $\left.d_{6}, 500 \mathrm{MHz}\right) \delta: 6.89,6.85$ (each $1 \mathrm{H}, \mathrm{d}, J$ $=2.2 \mathrm{~Hz}, \mathrm{~B}-3,5), 6.48(1 \mathrm{H}, \mathrm{d}, J=2.0 \mathrm{~Hz}, \mathrm{~A}-3), 6.30(1 \mathrm{H}, \mathrm{d}, J=2.0 \mathrm{~Hz}, \mathrm{~A}-5), 5.54(1 \mathrm{H}$, dd, $J=3.5,8.2 \mathrm{~Hz}$, glc-4), $5.27(1 \mathrm{H}, \mathrm{dd}, J=1.3,3.5 \mathrm{~Hz}$, glc-3), 5.08 (1H, ddd, $\mathrm{J}=3.4$, 4.2, 8.2 Hz, glc-5), $5.03(1 \mathrm{H}, \mathrm{dd}, J=1.3,5.9 \mathrm{~Hz}$, glc-2), $4.48(1 \mathrm{H}, \mathrm{d}, J=5.9 \mathrm{~Hz}$, glc-1), $4.23(1 \mathrm{H}, \mathrm{dd}, \mathrm{J}=3.4,12.4 \mathrm{~Hz}$, glc-6), $4.20(1 \mathrm{H}, \mathrm{dd}, J=4.2,12.4 \mathrm{~Hz}$, glc-6), 2.39, 2.26, 2.25, 2.13, 2.07, 2.02, 1.99, 1.98, 1.97 (each $\left.3 \mathrm{H}, \mathrm{s}, \mathrm{CH}_{3}\right) .{ }^{13} \mathrm{C}$ NMR (acetone- $d 6,125$ MHz) $\delta: 170.8,170.6,169.9,169.8,169.5,168.8,168.3,167.9,167.7$ (COO), 161.3 (A-2), 151.6 (A-4), 150.2, 149.9, 148.9 (B-2,4,6), 146.6 (A-6), 122.0 (B-1), 117.9 (A-1), 115.5, 113.3 (B-3,5), 107.8 (A-5), 101.1 (A-3), 88.8 (glc-2), 71.2 (glc-3), 69.8 (glc-4), 68.2 (glc-5), 61.3 (glc-6), 40.2 (glc-1), 21.1 (2C), 20.9, 20.8, 20.7, 20.6, 20.4, 20.3, 20.0 $\left(\mathrm{CH}_{3}\right)$.

\subsection{Reaction of phloroglucinol and dihydroxyacetone dimer}

Phloroglucinol (1.26 g) and dihydroxyacetone dimer (0.45 g) were dissolved in acetone $(5 \mathrm{~mL})$, and then the acetone was removed using a rotary evaporator. The resulting syrup was heated at $130^{\circ} \mathrm{C}$ for $1 \mathrm{~h}$, and then separated by silica gel column chromatography with $\mathrm{CHCl}_{3}: \mathrm{MeOH}: \mathrm{H}_{2} \mathrm{O}$ (90:10:1, 85:15:1, 80:20:2, 75:25:3, 70:30:5, and 50:50:0 v/v/v steps) to produce $12(356 \mathrm{mg}), 13(42 \mathrm{mg})$, phloroglucinol (348 $\mathrm{mg})$, and an oligomer fraction $(768 \mathrm{mg})$. A similar experiment using phloroglucinol (1.26 g) and methylglyoxal $(0.36 \mathrm{~g})$ yielded $12(686 \mathrm{mg})$ and recovered phloroglucinol (445 
$\mathrm{mg})$.

273

274

275

276

277

278

279

280

281

282

283

284

285

286

287

288

289

290

291

292

293

294

295

\subsubsection{Phloroglucinol dimer (12)}

Pale brown amorphous powder; UV $\lambda_{\max } \mathrm{nm}(\log \varepsilon)$ : 208 (4.47), 215 (4.61), 223 (4.05), 272 (0.62); IR $v_{\max } \mathrm{cm}^{-1}: 3242,1624$, 1487; FAB-MS (positive, matrix: glycerol) $m / z: 289[\mathrm{M}+\mathrm{H}]^{+}$; HR-FAB-MS m/z: $289.0710[\mathrm{M}+\mathrm{H}]^{+}\left(\right.$Calcd for $\mathrm{C}_{15} \mathrm{H}_{13} \mathrm{O}_{6}$ : 289.0712); ${ }^{1} \mathrm{H}$ NMR (acetone- $\left.d 6,500 \mathrm{MHz}\right) \delta: 5.99,5.98($ each $2 \mathrm{H}, \mathrm{d}, J=2.0 \mathrm{~Hz}$, $\left.\mathrm{H}-3,5,3^{\prime}, 5^{\prime}\right), 4.59(1 \mathrm{H}, \mathrm{s}, \mathrm{H}-7), 1.77$ (3H, s, H-9). ${ }^{13} \mathrm{C}$ NMR (acetone-d6, $\left.125 \mathrm{MHz}\right) \delta$ : 160.5, 160.2, 153.5 (each 2C, C-2,4,6,2',4',6'), 124.7 (C-8), 106.3 (2C, C-1, 1'), 96.8, 91.2 (each 2C, C-2, 4, 2', 4'), 30.6 (C-7), 24.6 (C-9).

\subsubsection{Phloroglucinol dimer (13)}

Pale brown powder; UV $\lambda_{\max } \mathrm{nm}(\log \varepsilon): 272$ (2.20); IR $v_{\max } \mathrm{cm}^{-1}: 3360,1631$, 1514, 1466, 1257, 1133, 928, 822, 452; ESI-MS (positive, matrix: glycerol) $\mathrm{m} / z: 289$ $[\mathrm{M}+\mathrm{H}]^{+}$; HR-FAB-MS m/z: $289.0713[\mathrm{M}+\mathrm{H}]^{+}$(Calcd for $\left.\mathrm{C}_{15} \mathrm{H}_{13} \mathrm{O}_{6}: 289.0712\right) ;{ }^{1} \mathrm{H}$ NMR (acetone- $d 6,500 \mathrm{MHz}) \delta: 6.34(1 \mathrm{H}, \mathrm{d}, J=7.9 \mathrm{~Hz}, \mathrm{H}-5 \mathrm{a}), 6.01(1 \mathrm{H}, \mathrm{d}, J=2.0 \mathrm{~Hz}$, H-9), $5.98(1 \mathrm{H}, \mathrm{d}, J=2.0 \mathrm{~Hz}, \mathrm{H}-7), 5.85(1 \mathrm{H}, \mathrm{d}, J=2.0 \mathrm{~Hz}, \mathrm{H}-2), 5.71(1 \mathrm{H}, \mathrm{d}, J=2.0$ Hz, H-4), 4.08 (1H, m, H-10b), 3.59 (1H, dd, $J=2.0,15.1 \mathrm{~Hz}, \mathrm{H}-11), 2.53$ (1H, dd, $J=$ 6.3, 15.1 Hz, H-11); ${ }^{13} \mathrm{C}$ NMR (acetone-d6, $125 \mathrm{MHz}$ ) $\delta: 162.0$ (C-6a), 159.5 (C-3), 157.3 (C-8), 156.3 (C-10), 155.6 (C-1), 155.2 (C-4a), 106.2 (C-5a), 105.4 (C-11a), 105.0 (C-10a), 98.2 (C-7), 96.6 (C-2), 89.7 (C-4), 40.8 (C-10b), 18.6 (C-11).

\section{Results and discussion}

\subsection{Pyrolysis of (-)-epigallocatechin-3-O-gallate}

Despite the high popularity of roasted green tea in Japan, only a few chemical studies have been published, and these studies have shown isomerization and 
oligomerization of tea catechins occur on roasting (Nakagawa, 1967; Hara \& Kubota, 1969; Anan, Amano, \& Nakagawa, 1981). However, the structures of the oligomers have not been studied. In this study, we examined pyrolysis of epigallocatechin-3-O-gallate (2) as a preliminary experiment. This catechin was selected because catechins with pyrogallol-type B-rings and galloyl esters account for over $70 \%$ and $60 \%$ of total tea catechins, respectively (Anan et al., 1981). Green tea leaves are typically roasted at $150-180^{\circ} \mathrm{C}$ (Nakagawa, 1967; Hara et al., 1969); therefore, a lyophilized powder of 2 was heated at $150^{\circ} \mathrm{C}$ for $60 \mathrm{~min}$ to afford (-)-gallocatechin-3-O-gallate, gallic acid, and an anthocyanidin together with recovered 2. The anthocyanidin was identified as tricetinidin, which was produced by elimination of gallic acid and subsequent oxidation. In this experiment, little or no oligomeric products were produced.

\subsection{Catechin oligomer from roasted green tea leaves}

Next, the commercial green tea leaves were heated at $180^{\circ} \mathrm{C}$ for $30 \mathrm{~min}$, and oligomeric polyphenols were obtained via solvent partitioning, adsorption column chromatography, and size-exclusion chromatography (Yanagida, Shoji, \& Shibusawa, 2003). Using HPLC analysis, the oligomeric products were detected as a broad hump on the baseline, and the UV absorption was similar to that of 2 (Fig. S1). The ${ }^{13} \mathrm{C} \mathrm{NMR}$ spectrum showed broad signals, which were closely related to those of 2 (Fig. 2A). However, the A-ring methine signals in the $\delta$ 95-100 range were much smaller than those of 2 (Fig. 2C). These signals shifted to lower field probably in the $\delta 105-115$ range, which suggests that oligomerization reactions occur at the A-ring methine carbons. In addition, broad signals in the $\delta 60-80$ range suggest sugars participate in the 
catechin oligomerization upon roasting.

\subsection{Catechin oligomer production from 2 and sucrose}

Because the most abundant sugar in tea leaves is sucrose, $\mathbf{2}$ was heated with sucrose at $150^{\circ} \mathrm{C}$ for $2 \mathrm{~h}$, and the resulting oligomers were separated (Fig. S2). The ${ }^{13} \mathrm{C}$ NMR spectrum (Fig. 2B) was similar to that of the oligomer fraction obtained from roasted green tea leaves except for the absence of the catechol-type B-ring originating from $\mathbf{3}$ and $\mathbf{4}$. These results confirmed the cross-linking of $\mathbf{2}$ with the sugars.

\subsection{Catechin oligomer produced from 2 and ${ }^{13} \mathrm{C}$-labeled glucose}

Next, the oligomers generated upon heating $\left(150^{\circ} \mathrm{C}\right.$ for $\left.2 \mathrm{~h}\right)$ of $\mathbf{2}$ with glucose were examined because pyrolysis of sucrose upon roasting produces glucose and fructose as major products ( $\mathrm{S}^{\sim}$ imkovic, S`urina, \& Vric $`$ an, 2003). The ${ }^{13} \mathrm{C}$ NMR spectrum of the oligomeric polyphenols obtained upon heating of $\mathbf{2}$ with glucose (Fig. 3 A) was similar to those of oligomers of roasted green tea leaves and oligomers prepared from 2 and sucrose (Fig. 2). The co-oligomerization was clearly confirmed by experiments using $1{ }^{13} \mathrm{C}$-labeled glucose (Fig. 3B) and $\mathrm{U}-{ }^{13} \mathrm{C} 6$-labeled glucose (Fig. 3C). The spectrum of the oligomer obtained upon heating with $1-{ }^{13} \mathrm{C}$-labeled glucose exhibited large signals at $\delta 100$ and $\delta 33$ (Fig. 3B, black arrows), which were attributed to the carbons of the glucose anomeric carbon. The signal at $\delta 100$ may be explained by formation of the $O$-glycosidation linkage at the anomeric position. In the spectrum of the oligomer obtained upon heating of the $\mathrm{U}^{13} \mathrm{C} 6$-labeled glucose, signals at $\delta 115$ and from $\delta 60$ to 84 (Fig. 3C, black arrows) were enhanced in addition to the $\delta 100$ and $\delta 33$ signals. The large signals between $\delta 60$ and 84 were attributed to glucose C-2 to C-6 
carbons. Enhancement of the signals at $\delta 115$ and 33 upon incorporation of the ${ }^{13} \mathrm{C}$ was likely related to the oligomerization mechanism.

\subsection{Monomeric and dimeric products of 2 produced upon heating with glucose}

To understand the oligomerization mechanisms, lyophilized powder of a mixture of 2 and D-glucose was heated at $150^{\circ} \mathrm{C}$ for a shorter period (30 min), and the products were separated by column chromatography to yield four products. Products $\mathbf{5}$ and 6 were a pair of isomers of glucose C-glucosides according to FAB-MS, which had a $[\mathrm{M}+\mathrm{H}]^{+}$peak at $m / z 621$. The ${ }^{1} \mathrm{H}$ and ${ }^{13} \mathrm{C}$ NMR spectra showed signals arising from 2 and glucopyranose moieties; however, the A-ring proton signals were observed as one proton singlet signal in each spectrum [5: $5.98(\mathrm{~s}, \mathrm{H}-6), 6: 6.06(\mathrm{~s}, \mathrm{H}-8)]$, and the glucose C-1 of 5 and $\mathbf{6}$ resonated at $\delta 76.2$ and $\delta 76.8$, respectively (Fig. S3). These data confirmed that glucose was attached to the A-ring C-8 or C-6 of 2 via C-glycosidic linkages. The HMBC spectrum of $\mathbf{6}$ showed the correlation of the A-ring C-8a with the A-ring $\mathrm{H}-8$ and $\mathrm{C}$-ring $\mathrm{H}-2$, indicating that the glucose of 6 was located at the C-6 position (Fig. S3). Thus, we concluded the glucose in $\mathbf{5}$ was located at C-8. From these spectroscopic data, 5 and $\mathbf{6}$ were determined to be epigallocatechin-3-O-gallate 8-C-glucoside and 6-C-glucoside, respectively (Fig. 4).

The ${ }^{1} \mathrm{H}$ and ${ }^{13} \mathrm{C}$ NMR spectra of the other monomeric product, 7 , showed a set of signals of $\mathbf{2}$ and two sets of signals for the sugar moiety. The absence of the A-ring methine proton signal in the ${ }^{1} \mathrm{H}$ NMR spectrum and the molecular formula of $\mathrm{C}_{34} \mathrm{H}_{37} \mathrm{O}_{20}$, which was confirmed by HR-ESI-MS $\left([\mathrm{M}+\mathrm{H}]^{+} \mathrm{m} / \mathrm{z}\right.$ 765.1873, Calcd for $\mathrm{C}_{34} \mathrm{H}_{37} \mathrm{O}_{20}$ : 765.1873), both indicated that 7 was a disubstituted analog of 5 and $6 .{ }^{1} \mathrm{H}$ and ${ }^{13} \mathrm{C}$ signals arising from one of the two sugar units were similar to the signals of 
C-glycosidic glucopyranose moieties of $\mathbf{5}$ and 6. Another sugar unit showed signals that were assignable to two methylenes ( $\delta 34.8, \mathrm{C}-1 " ' ;$ and $\left.61.4, \mathrm{C}-6{ }^{\prime \prime}\right)$, three oxygenated methines ( $\delta$ 76.6, C-4"'; 80.7, C-3"'; and 83.9, C-5"'), and an acetal quaternary carbon $(\delta$ 116.9, C-2"'). The HMBC correlations (Fig. 5) and unsaturation index of this molecule (17) suggested the presence of a spiroketal structure involving an ether linkage with an A-ring phenolic hydroxy group. The location of the hydroxy group participating in the acetal ring formation was determined by observation of hydrogen-deuterium exchange shifts of the A-ring carbons (Pfeffer, Valentine, \& Parrish, 1979). The ${ }^{13} \mathrm{C}$ NMR spectra of 7 measured in acetone- $d_{6}+\mathrm{H}_{2} \mathrm{O}$ and acetone- $d_{6}+\mathrm{D}_{2} \mathrm{O}$ were carefully compared, and a distinct chemical shift difference was observed for C-7 $(\Delta \delta+0.123)$. By contrast, the shifts for C-5 $(\Delta \delta-0.008)$ and $\mathrm{C}-8 \mathrm{a}(\Delta \delta+0.025)$ were minimal (Fig. S4). This observation indicated the presence of a free hydroxy group at the A-ring C-7. Because the sugar units originated from D-glucose, the configuration of the sugar carbons was self-evident except for the spiroketal carbon. We deduced the spiroketal was in the $S$-configuration from the NOE between the C-1 and C-3 protons. Based on these results, the structure of 7 was determined (Fig. 4). The chemical shifts of the spiroketal carbon $(\mathrm{C}-2$ '", $\delta 116.9)$ and benzylic methylene carbon $\left(\mathrm{C} 1{ }^{\prime \prime}, \delta 34.8\right)$ coincided with the values of enhanced carbon signals ( $\delta 115$ and 33) in the spectra of the oligomers obtained upon condensation with the ${ }^{13} \mathrm{C}$-labeled glucoses (Fig. 3). Therefore, a similar spiroketal structure may be present in the oligomeric products. for $\mathrm{C}_{50} \mathrm{H}_{42} \mathrm{NaO}_{25}$ : 1065.1913), product 8 is a dimeric product. The ${ }^{1} \mathrm{H}$ and ${ }^{13} \mathrm{C} \mathrm{NMR}$ spectra showed that a sugar residue connected two epigallocatechin gallate moieties. 
392

393

394

395

396

397

398

399

400

401

402

403

404

405

406

407

408

409

410

411

412

413

414

415

methine carbons (C-6 or C-8) were attached to the sugar residue. The sugar residue was composed of six carbons: a trisubstituted double bond ( $\delta 148.2, \mathrm{C}-1 ; \delta 101.7, \mathrm{C}-2)$, a non-oxygenated methine carbon $(\delta 38.1, \mathrm{C}-3)$, two oxygenated methines $(\delta 74.0, \mathrm{C}-4 ; \delta$ 74.4, C-5), and an oxygenated methylene carbon ( $\delta 64.3, \mathrm{C}-6)$. Formation of a pyran was deduced from the unsaturation index (30), and ${ }^{1} \mathrm{H}-{ }^{1} \mathrm{H}$ COSY and HMBC correlations of the sugar protons with the A-rings of epigallocatechin units showed a dimeric structure (Fig. 4). It was acceptable that the 4-, 5-, and 6-positions of the sugar moiety retained the configuration of the D-glucose, and the 3,4-cis configuration was suggested by the small $J_{2,3}$ value $(3.0 \mathrm{~Hz})$. However, we could not determine where the sugar residue attached to catechin C-6 or C-8 because of the lack of the HMBC correlations from the catechin C-ring $\mathrm{H}-2$ to the A-ring C-8a; therefore, the structure in Fig. 4 is a tentative one. A plausible production mechanism of $\mathbf{8}$ is proposed in Scheme S1, which explains the catechin cross-linking with the sugar. Similar reactions may contribute to oligomerization of $\mathbf{2}$ in roasted tea, although it was difficult to identify the signals assignable to the sugar moiety of $\mathbf{8}$ in the ${ }^{13} \mathrm{C}$ NMR spectra of the oligomers (Fig. $3)$.

\subsection{Reaction of phloroglucinol with glucose and its pyrolysis products}

In addition to the low-field shifts of the A-ring methine carbon signals in the ${ }^{13} \mathrm{C}$ NMR spectra of the oligomeric products (Figs. 2 and 3), the results of the abovementioned chemical examinations indicated that the A-rings were responsible for the catechin oligomerization. To investigate the reaction mechanism in more detail, we selected phloroglucinol as a simple A-ring mimic and examined the reactions with glucose. Heating of phloroglucinol with glucose afforded C-glycoside 9 (Onodera, 
Yamamoto, Abe, \& Ueno, 1994) and two new dimeric products, 10 and 11 (Fig. 6). The structure of product $\mathbf{1 0}$ was determined by ${ }^{1} \mathrm{H}-{ }^{1} \mathrm{H}$ COSY, HSQC, and HMBC experiments (Fig. S5). Dimer $\mathbf{1 1}$ was obtained as an inseparable mixture of stereoisomers. The major component of this mixture was purified as an acetate, and its structure was confirmed (Fig. S6). In the ${ }^{13} \mathrm{C}$ NMR spectrum of $\mathbf{1 0}$, the $\mathrm{C}-1$ of the glucose moiety resonated at $\delta 33.5$, and the chemical shift was similar to one of the enhanced carbon signals of the oligomers obtained in the experiments using the

${ }^{13} \mathrm{C}$-labeled glucoses (Fig. 3). The structures of $\mathbf{1 0}$ and $\mathbf{1 1}$ suggested another mechanism for the cross-linking of $\mathbf{2}$ with the sugars in addition to that suggested by the production of 8 (Scheme S2).

Reactions with the pyrolysis products of the sugars should also be considered

because heating of sucrose, the most abundant sugar in tea leaves, is known to afford glucose and fructose, and further degradation of the monosaccharides generates methyl glyoxal (MG) and dihydroxyacetone (DHA) (Kabyemela, Adschiri, Malaluan, \& Ara, 1997). Both MG and DHA are also generated in the Maillard reaction (Totlani \& Peterson, 2006). In addition, MG is produced from DHA. In this study, phloroglucinol was heated separately with MG and the DHA dimer, and $\mathbf{1 2}$ was obtained as a major product in the reaction with MG. Products 12 and 13 were obtained from the reaction with DHA. The structures were determined by spectroscopic methods and computer calculations (Figs. S7 and S8). Production of 12 via both reactions supported the conversion of DHA to MG upon heating. These reactions also afforded oligomeric products. The ${ }^{13} \mathrm{C}$ NMR spectrum of the oligomeric products obtained by heating phloroglucinol with $2-{ }^{13} \mathrm{C}$-labeled DHA (Fig. S9) showed broad signals at $\delta 124$ (enhanced by incorporation of ${ }^{13} \mathrm{C}$ ), $\delta 48$, and $\delta 24$, which coincided with the C-8 $(\delta$ 

oligomerization mechanism of phloroglucinol with DHA is similar to the production mechanism of 12 (Scheme S3). Furthermore, the broad signal observed in the $\delta$ 105-115 range is assignable to aromatic carbons where the MG unit connected. In the

${ }^{13} \mathrm{C}$ NMR spectrum of the oligomer obtained from the roasted tea (Fig. 2), signals for the catechin $\mathrm{C} 6$ and $\mathrm{C} 8$ carbon that were involved in the oligomerization reaction were likely present in a similar region.

\section{Conclusions}

In this study, we showed that roasting green tea leaves causes epimerization of the catechin C-ring and co-oligomerization of the catechin with sugar or its pyrolysis products. The co-oligomerization occurs at the catechin A-ring, which is shown by low-field shifts of the A-ring methine carbon signals from their original locations at $\delta$ 95-98 to $\delta 105-115$. Structures of the products obtained via model reactions using 2 and its A-ring mimic, phloroglucinol, suggest that the oligomerization of catechins does not proceed uniformly; however, the basic mechanism involves cross-linking at the A-ring C6 and/or C8 with the carbonyl carbons of sugar or its pyrolysis products. The roasting process decreases the astringency caused by tea catechins and the astringency of the oligomer fraction obtained from roasted tea is milder than that of the original green tea catechins. A similar reaction should occur during roasting of cacao and other catechinand proanthocyanidin-containing foods. Evaluation of the biological functions of the oligomers is now in progress.

\section{Conflict of interest}


The authors declare that they have no conflicts of interest.

465

466

467

468 for editing a draft of this manuscript.

472

Appendix A. Supplementary data

Supplementary data associated with this article can be found, in the online version, at https://doi.org/

\section{References}

Anan, T., Amano, I, \& Nakagawa, M. (1981). Changes in contents of some ingredients during heating of green tea. Journal of Japanese Society of Food Science and Technology, 28, 74-78.

Baxter, N. J., Lilley, T. H., Haslam, E., \& Williamson, M. P. (1997). Multiple interactions between polyphenols and a salivary proline-rich protein repeat result in complexation and precipitation. Biochemistry, 36, 5566-5577.

Coggon, P., Moss, G. A., Graham, H. N., \& Sanderson, G. W. (1973). Biochemistry of tea fermentation. Oxidative degallation and epimerization of the tea flavanol gallates. Journal of Agricultural and Food Chemistry, 21, 727-733.

De Taeye, C., Bodart, M., Caullet, G., \& Sonia Collin, S. (2017). Roasting conditions 
for preserving cocoa flavan-3-ol monomers and oligomers: interesting behavior of Criollo clones. Journal of the Science of Food and Agriculture, 97, 4001-4008.

490

491

492

493

Hara, T. \& Kubota, E. (1969) Effects of roasting condition on the qualities of roasted green tea. Journal of Japanese Society of Food Science and Technology, 16, 145-149.

Haslam, E. (1996). Natural polyphenols (vegetable tannins) as drugs: possible modes of action, Journal of Natural Products, 59, 205-215.

Ishii, T., Minoda, K., Bae, M.-J., Mori, T., Uekusa, Y., Ichikawa, T., Aihara, Y., Furuta, T., Wakimoto, T., Kan, T., \& Nakayama, T. (2010). Binding affinity of tea catechins for HSA: Characterization by high-performance affinity chromatography with immobilized albumin column, Molecular Nutrition \& Food Research, 54, 816-822.

Kabyemela, B. M., Adschiri, T., Malaluan, R. M., \& Arai, K. (1997). Kinetics of glucose epimerization and decomposition in subcritical and supercritical water, Industrial \& Engineering Chemistry Research, 36, 1552-1558.

Kuhnert, N., Clifford, M. N., \& Radenac, A-G. (2001). Boron trifluoride-etherate mediated synthesis of 3-desoxyanthocyanidins including a total synthesis of tricetanidin from black tea. Tetrahedron Letters, 42, 9261-9263 (2001)

Moreira, A. S. P., Nunes, F. M., Simões, C., Maciel, E., Domingues, P., Domingues, M. R. M., \& Coimbra, M. A. (2017). Transglycosylation reactions, a main mechanism of phenolics incorporation in coffee melanoidins: Inhibition by Maillard reaction, Food Chemistry, 227. 422-431.

Nakagawa, M. (1967). The nature and the origin of polyphenols in Hoji-cha (roasted green tea), Agricultural and Biological Chemistry, 31, 1283-1287.

Nonaka, G., Kawahara, O., \& Nishioka, I. (1983) Tannins and related compounds. XV. 
A new class of dimeric flavan-3-ol gallates, theasinensins $\mathrm{A}$ and $\mathrm{B}$, and proanthocyanidin gallates from green tea leaf. (1), Chemical \& Pharmaceutical Bulletin, 31, 3906-3914.

Onodera, J., Yamamoto, T., Abe, K., \& Ueno, N. (1994). Cosmetics containing phloroglucinol derivatives, Jpn. Kokai Tokkyo Koho, JP 06092835 A 19940405.

Pfeffer, P. E., Valentine, K, M., \& Parrish, F. W. (1979). Deuterium-induced differential isotope shift carbon-13 NMR. 1. Resonance reassignments of mono- and disaccharides. Journal of the American Chemical Society, 101, 1265-1274.

S`imkovic, I., S`urina, I., \& Vric`an, M. (2003). Primary reactions of sucrose thermal degradation, Journal of Analytical and Applied Pyrolysis, 70, 493-504.

Seto, R., Nakamura, H., Nanjo, F., \& Hara, Y. (1997). Preparation of epimers of tea catechins by heat treatment, Bioscience, Biotechnology, and Biochemistry, 61, $1434-1439$.

Suzuki, M., Sano, M., Yoshida, R., Degawa, M., Miyase, T., \& Maeda-Yamamoto, M. (2003). Epimerization of tea catechins and $O$-methylated derivatives of (-)-epigallocatechin-3-O-gallate: relationship between epimerization and chemical structure, Journal of Agricultural and Food Chemistry, 51, 510-514.

Totlani, V. M. \& Peterson, D. G. (2006). Epicatechin carbonyl-trapping reactions in aqueous Maillard systems: identification and structural elucidation, Journal of Agricultural and Food Chemistry, 54, 7311-7318.

Yanagida, A., Shoji, T., \& Shibusawa, Y. (2003). Separation of proanthocyanidins by degree of polymerization by means of size-exclusion chromatography and related techniques. Journal of Biochemical and Biophysical Methods, 56, 311-322. 


\section{Figure Captions}

538 Fig. 1. HPLC profiles (max abs) of extracts of green tea leaves and roasted green tea 539 leaves: A, Extract of green tea leaves; B, extract of roasted green tea leaves $(0.02 \mathrm{~g} / \mathrm{mL}$ 540 in $60 \% \mathrm{EtOH})$; and $\mathrm{C}$, vertical axis expansion of B. Compounds: 1, 541 (-)-epigallocatechin; 2, (-)-epigallocatechin-3-O-galalte; 3, (-)-epicatechin; 4, 542 (-)-epicatechin-3-O-galalte; 1a, (-)-gallocatechin; 2a, (-)-gallocatechin-3-O-galalte; 3a, 543 (-)-catechin; 4a, (-)-catechin-3-O-galalte; GA, gallic acid; and caf, caffeine.

545 Fig. $2{ }^{13} \mathrm{C}-\mathrm{NMR}$ spectra (in DMSO- $d_{6}$ ) of oligomeric polyphenols obtained from roasted green tea leaves $\left(180^{\circ} \mathrm{C}\right)(\mathrm{A})$, oligomer obtained upon heating of 2 with sucrose (B), and 2 (C). Labeling: A, A-ring; B, B-ring; C, C-ring; G, galloyl; and

Fig. 3. ${ }^{13} \mathrm{C}-\mathrm{NMR}$ spectra of the oligomer fraction obtained upon heating of 2 with glucose (A), $1-{ }^{13} \mathrm{C}$-labeled glucose (B), and $\mathrm{U}^{13} \mathrm{C} 6$-labeled glucose (C). The black arrows indicate signals enhanced by incorporation of ${ }^{13} \mathrm{C}$.

553

Fig. 4. Structures of 5-8 produced upon heating of $\mathbf{2}$ with glucose.

Fig. 5. Selected ${ }^{1} \mathrm{H}-{ }^{1} \mathrm{H}$ COSY and HMBC correlations of 7.

Fig. 6. Products generated from phloroglucinol with glucose (9-11), methyl glyoxal 

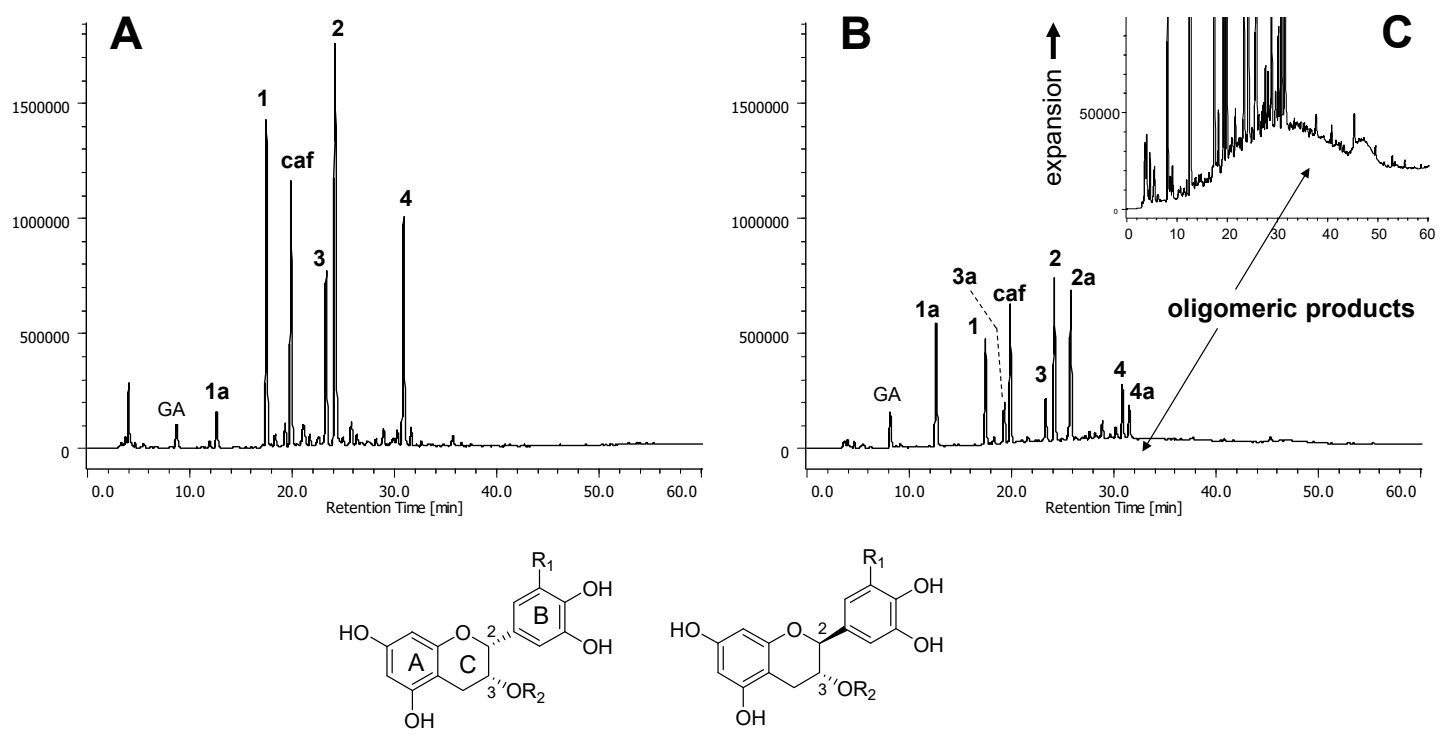

565
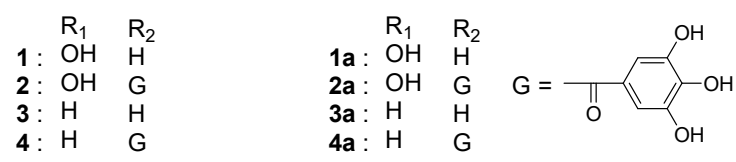

566

567 Fig. 1. HPLC profiles (max abs) of extracts of green tea leaves and roasted green tea 568 leaves.

569 A: Extract of green tea leaves, B: extract of roasted green tea leaves $(0.02 \mathrm{~g} / \mathrm{mL} 60 \%$ 570 EtOH). C: vertical axis expansion of B. 1: (-)-epigallocatechin, 2: 571 (-)-epigallocatechin-3-O-galalte, 3: (-)-epicatechin, 4: (-)-epicatechin-3-O-galalte, 1a:

572 (-)-gallocatechin, 2a: (-)-gallocatechin-3-O-galalte, $\quad 3 \mathbf{a}: \quad(-)$-catechin, $\quad 4 a:$ 573 (-)-catechin-3-O-galalte, GA: gallic acid, caf: caffeine. 


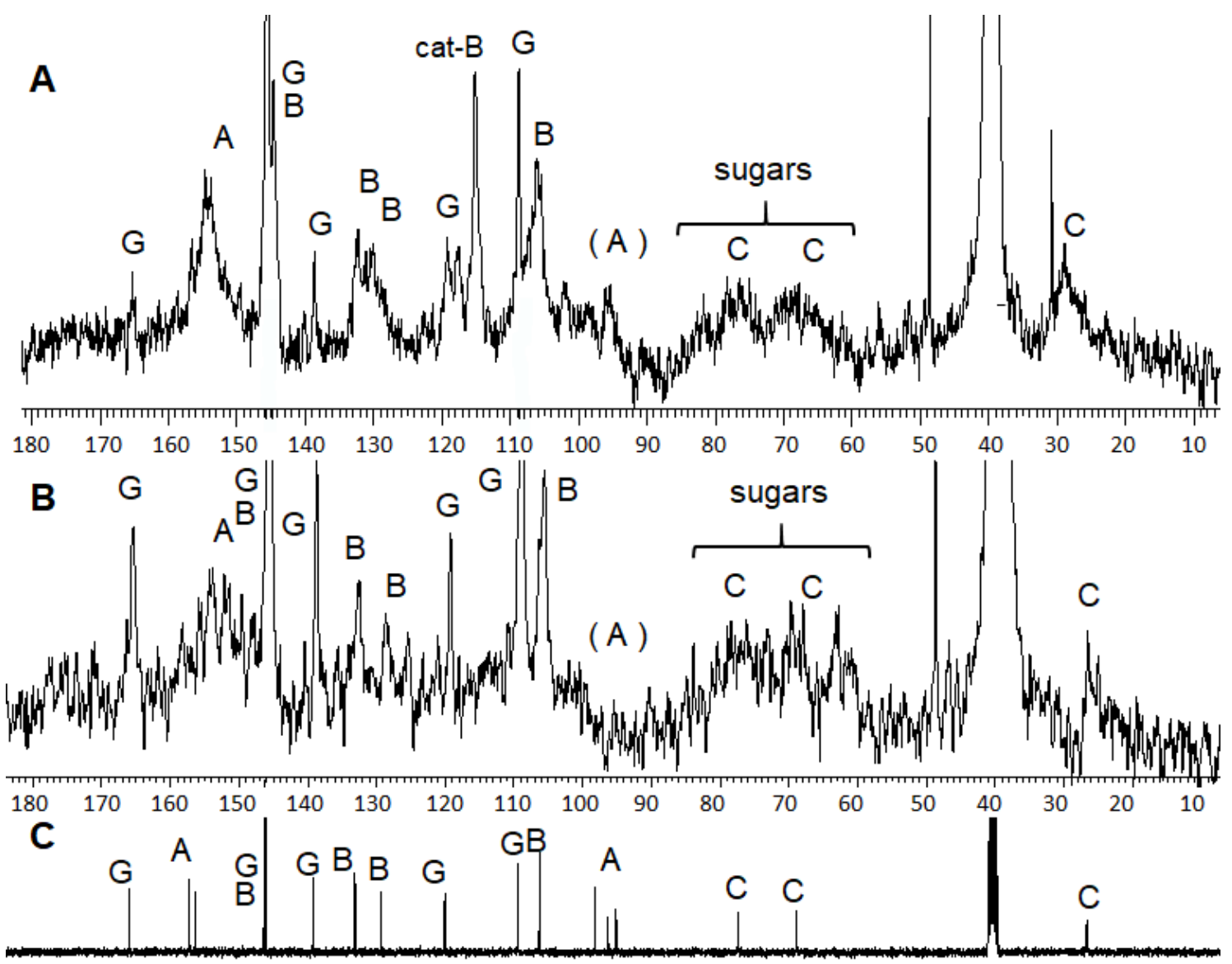

ता

579 Fig. $2{ }^{13} \mathrm{C}-\mathrm{NMR}$ spectra (in DMSO- $d_{6}$ ) of oligomeric polyphenols obtained from

580 roasted green tea leaves $\left(180^{\circ} \mathrm{C}\right)(\mathrm{A})$, oligomer obtained upon heating of 2 with sucrose 581 (B), and 2 (C). A: A-ring, B: B-ring, C: C-ring, G: galloyl, Cat-B: catechol-type B-ring. 


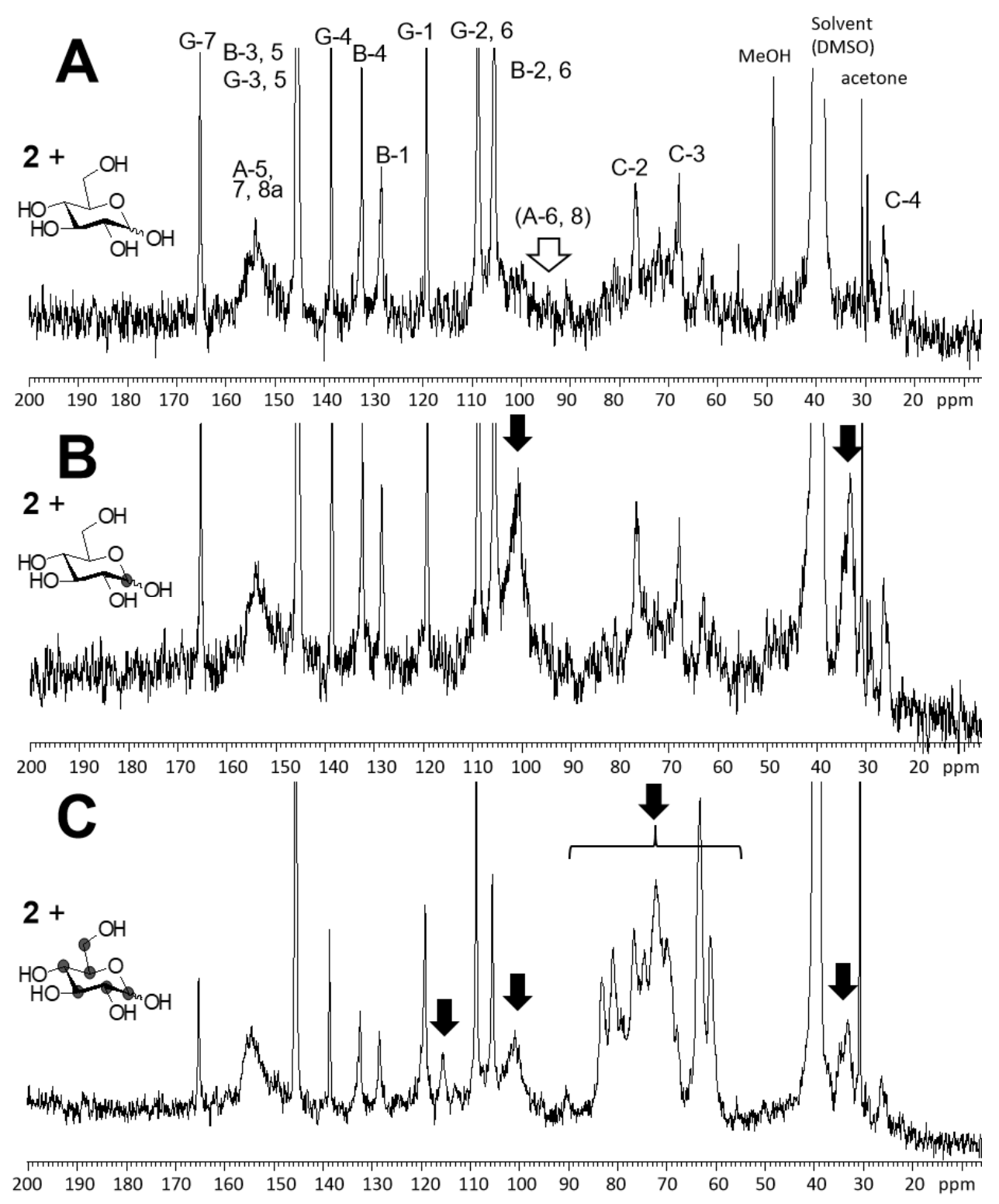

Fig. 3. ${ }^{13} \mathrm{C}$-NMR spectra of oligomer fractions obtained upon heating of 2 with glucose (A), $1-{ }^{13} \mathrm{C}$-labeled glucose (B) and $\mathrm{U}^{13} \mathrm{C} 6$-labeled glucose (C). The black arrows indicate signals enhanced by incorporation of ${ }^{13} \mathrm{C}$. 
596<smiles>O=C(O)COC(O)COC1Cc2c(O)cc(O)c(OC(=O)c3cc(O)c(O)c(O)c3O)c2OC1c1cc(O)c(O)c(O)c1</smiles><smiles>O=C(O)COC(O)COc1cc(O)c(O)c(OC(=O)c2cc(O)c(O)c(O)c2)c1C1Oc2cc(O)c(O)c(O)c2CC1O</smiles>

6

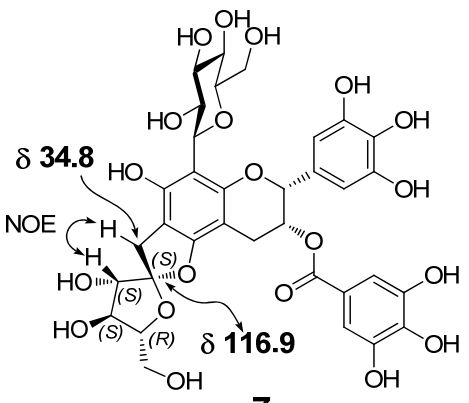

597

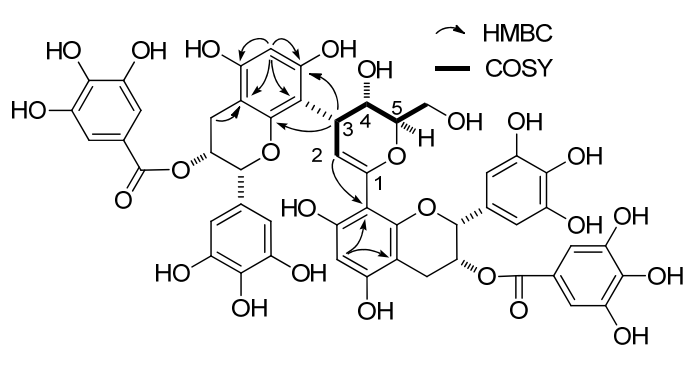

8

598

599

Fig. 4. Structures of 5-8 produced upon heating 2 with glucose.

600

601

602

603 


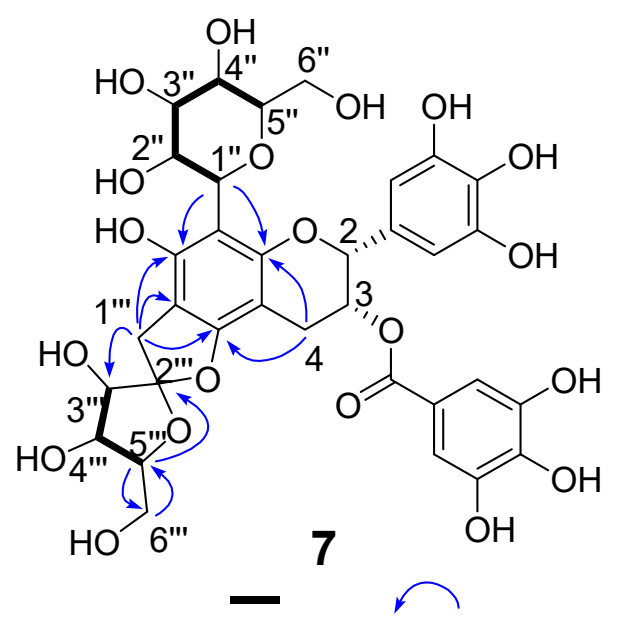

607

608 Fig. 5. Selected ${ }^{1} \mathrm{H}-{ }^{1} \mathrm{H}$ COSY and HMBC correlations of 7.

609

610

611

612 
616<smiles>Oc1cc(O)c(C(O)O)c(O)c1</smiles>

9

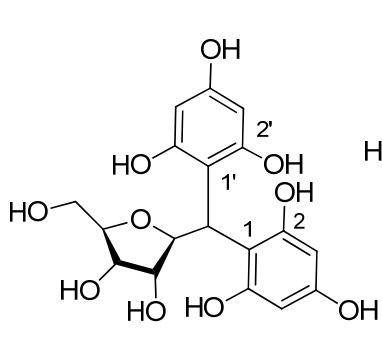

10

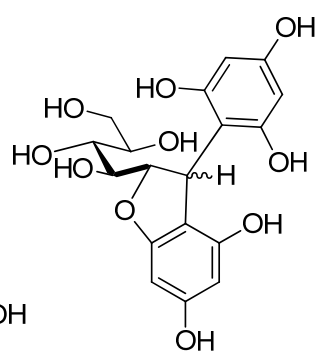

11

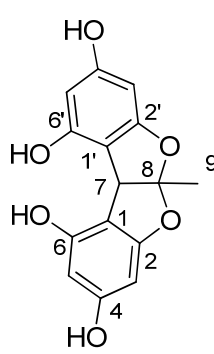

12

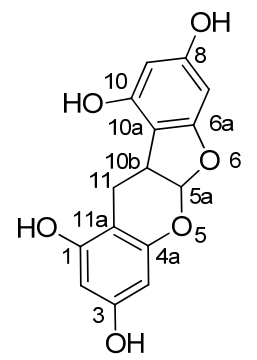

13

618

619 Fig. 6. Products generated from phloroglucinol with glucose (9-11), with methyl 620 glyoxal (12), and with dihydroxyacetone (12 and 13).

621

622

623

624 